\title{
Toward point-of-care assessment of patient response: a portable tool for rapidly assessing cancer drug efficacy using multifrequency impedance cytometry and supervised machine learning
}

\author{
Karan Ahuja', Gulam M. Rather $\mathbb{D}^{2}$, Zhongtian Lin' ${ }^{1}$, Jianye Sui ${ }^{1}$, Pengfei Xie ${ }^{1}$, Tuan Le ${ }^{1}$, Joseph R. Bertino ${ }^{2}$ and \\ Mehdi Javanmard ${ }^{1}$
}

\begin{abstract}
We present a novel method to rapidly assess drug efficacy in targeted cancer therapy, where antineoplastic agents are conjugated to antibodies targeting surface markers on tumor cells. We have fabricated and characterized a device capable of rapidly assessing tumor cell sensitivity to drugs using multifrequency impedance spectroscopy in combination with supervised machine learning for enhanced classification accuracy. Currently commercially available devices for the automated analysis of cell viability are based on staining, which fundamentally limits the subsequent characterization of these cells as well as downstream molecular analysis. Our approach requires as little as $20 \mu \mathrm{L}$ of volume and avoids staining allowing for further downstream molecular analysis. To the best of our knowledge, this manuscript presents the first comprehensive attempt to using high-dimensional data and supervised machine learning, particularly phase change spectra obtained from multi-frequency impedance cytometry as features for the support vector machine classifier, to assess viability of cells without staining or labelling.
\end{abstract}

\section{Introduction}

Cancer continues to be one of the leading causes of death worldwide. The primary treatment options for cancer include surgery ${ }^{1}$, chemotherapy ${ }^{2}$, radiation therapy $^{3}$, hormonal therapy ${ }^{4}$, targeted therapy ${ }^{5}$, and palliative care $^{6}$. The choice of therapy mainly depends upon the type and stage of cancer, legal issues, clinical infrastructure, past response rates, and the patient's health conditions. Chemotherapy is non-specific and results in killing non-targeted cells, which results in many side effects including hair loss and serious gastrointestinal

\footnotetext{
Correspondence: Mehdi Javanmard (mehdi.javanmard@rutgers.edu) ${ }^{1}$ Department of Electrical and Computer Engineering, Rutgers University, New Brunswick, NJ, USA

${ }^{2}$ Rutgers Cancer Institute of New Jersey, Rutgers University, New Brunswick, NJ, USA
}

issues. A targeted approach, where only tumor cells are eliminated, with minimal effect on non-tumor cells would result in higher efficacy with minimal side effects. New therapeutic agents as well as diagnostic tools predicting patient response are urgently needed. In this work we present a novel method to rapidly assess drug efficacy in targeted cancer therapy, where antineoplastic agents are conjugated to antibodies targeting surface markers on tumor cells. "Activated" matriptase, a membrane-bound protease, is overexpressed in various epithelial cancer cells, including B-cell lymphoma, multiple myeloma, and epithelial carcinomas ${ }^{7,8}$. Given the importance of matriptase in tumor behavior and its expression on a wide variety of tumor cell types, the targeted delivery of cancer drugs to the tumor site shows great promise for enhancing drug efficacy and minimizing toxicity toward

\section{(c) The Author(s) 2019}

(c) (i) Open Access This article is licensed under a Creative Commons Attribution 4.0 International License, which permits use, sharing, adaptation, distribution and reproduction cc) in any medium or format, as long as you give appropriate credit to the original author(s) and the source, provide a link to the Creative Commons license, and indicate if changes were made. The images or other third party material in this article are included in the article's Creative Commons license, unless indicated otherwise in a credit line to the material. If material is not included in the article's Creative Commons license and your intended use is not permitted by statutory regulation or exceeds the permitted use, you will need to obtain permission directly from the copyright holder. To view a copy of this license, visit http://creativecommons.org/licenses/by/4.0/. 
noncancerous cells. Cell sensitivity to drug can be assessed by qualitatively analyzing the surface markers on the tumor cells to determine whether the targeted therapy will be effective or not. Cells sensitive to the antimatriptase-conjugated drug will undergo apoptosis (cell death), while the cells which are insensitive to the drug will remain alive. We have fabricated and characterized a device capable of rapidly assessing cancer cell viability in response to anti-matriptase-conjugated drugs using multifrequency impedance spectroscopy in combination with machine learning for enhanced classification accuracy, without the need for any staining or labeling of the cells.

The gold standard for automated cell viability analysis is the Vi-Cell instrument developed by Beckman Coulter ${ }^{\circ}$. It uses the trypan blue dye exclusion method to perform analysis of cell viability. Staining fundamentally limits the subsequent characterization of cells as well as downstream molecular analysis and requires bulky optical instrumentation to assess the viability. In addition, this method requires $0.5-1 \mathrm{~mL}$ of sample volume. In addition, various assays are available for measuring different markers which indicate cell death (cytotoxicity assay), the mechanism of cell death, and the quantity of live cells (viability assay) $)^{9,10}$. Drug screening platforms utilizing nano and microfluidic channels have been widely explored $^{11-19}$. The use of microfluidic channels can help reduce sample volumes and the cost of reagents. Recently droplet microfluidics has been effectively used for drug screening of cancer cell lines ${ }^{20}$. Apart from microfluidic techniques, optical coherence tomography (OCT) has been used to track cell death ${ }^{21}$. Although OCT is label free and can quantitatively track cell death, the use of optical techniques requires bulky optical instrumentation, making it less compatible with the needs of point-of-care. Screening cells based on their electrical properties can enable label-free assessment of cell viability. The use of dielectropheresis has been vastly explored to assess cell viability, growth, and immuno-reactivity ${ }^{22,23}$. The primary drawback of dielectropheresis is that for each cell, sorting/ analysis based on dielectric properties is performed only at a single frequency. The use of multiple frequencies simultaneously can provide a snapshot of a cell's dielectric properties over a wide range of frequencies, resulting in higher classification accuracy.

Electrical impedance spectroscopy/cytometry enables measuring AC electrical properties of particles in suspension through which the frequency dependent dielectric parameters of the particles can be obtained. The primary advantage of impedance cytometry is that it is label free, and analysis can be performed at a single cell level. The use of bio-impedance measurements can date back to the early 20th century ${ }^{24,25}$ where low and high frequency conductivity of erythrocytes were measured. Since then, there have been many advancements in the field of microfluidic single cell impedance analysis ${ }^{26}$. Microfluidic impedance cytometry has shown promising results in various fields such as analysis and differentiation of leukocytes ${ }^{27}$ and platelets ${ }^{28}$, whole blood cell differentiation ${ }^{29}$, nano-electronic barcoding of particles ${ }^{30}$, tumor cell characterization and classification ${ }^{31}$, and PicoMolar level detection of protein biomarkers ${ }^{32}$, among other fields. In the past, multifrequency impedance cytometry has also been used to analyze membrane potential and viability of Bacillus megaterium cells ${ }^{33}$. Although multifrequency impedance cytometry was used, only one frequency $(10 \mathrm{MHz})$ was used to analyze cell viability of Bacillus megaterium cells. One frequency may not be sufficient to accurately classify whether a cell is live or dead. We extend the advancements made in impedance cytometry toward a new direction where analysis of cell viability of the cancer cells is based on their impedance response in conjunction with machine learning for enhanced classification accuracy.

A field of computer science that enables computers to learn without explicitly being programmed is machine learning. Evolved from the study of pattern recognition and computational learning theory in artificial intelligence, machine learning plays a pivotal role in the study and construction of algorithms, which learn and make predictions on data. Support vector machines (SVMs) are supervised learning models that have algorithms associated with them to analyze data for classification and regression ${ }^{34}$. SVM's are efficient in performing nonlinear classification, implicitly mapping their inputs to highdimensional feature spaces. Machine learning has found wide applications within biology and bioinformatics to make accurate predictions ${ }^{35-37}$. We demonstrate the use of machine learning toward building a portable point-ofcare diagnostic tool for assessing patient response to targeted cancer therapy. A patient's cancer cells are treated with antibody-conjugated drugs, and the proposed impedance cytometer determines the percentage of live and dead tumor cells in the sample. A larger proportion of dead cells in the sample is indicative that the cells express activated matriptase and that the targeted therapy will be effective.

Figure 1 illustrates the schematic diagram of the system. This includes a microfluidic channel embedded on a glass wafer with gold electrodes (Fig. 2), a multifrequency lock in amplifier (Zurich Instruments ${ }^{\circledR}$ ), and software to record and analyze the data. Figure $2 \mathrm{a}$ represents microfabricated electrodes integrated in the channel and Fig. $2 \mathrm{~b}$ represents cancer cells (T47D) flowing through the microfluidic channel. Impedance cytometry experiments were conducted after the fabrication of devices. In this work we conducted experiments with T47D cancer cells, T47D cancer cells treated with target drug and T47D dead cancer cells. We conducted the impedance cytometry 


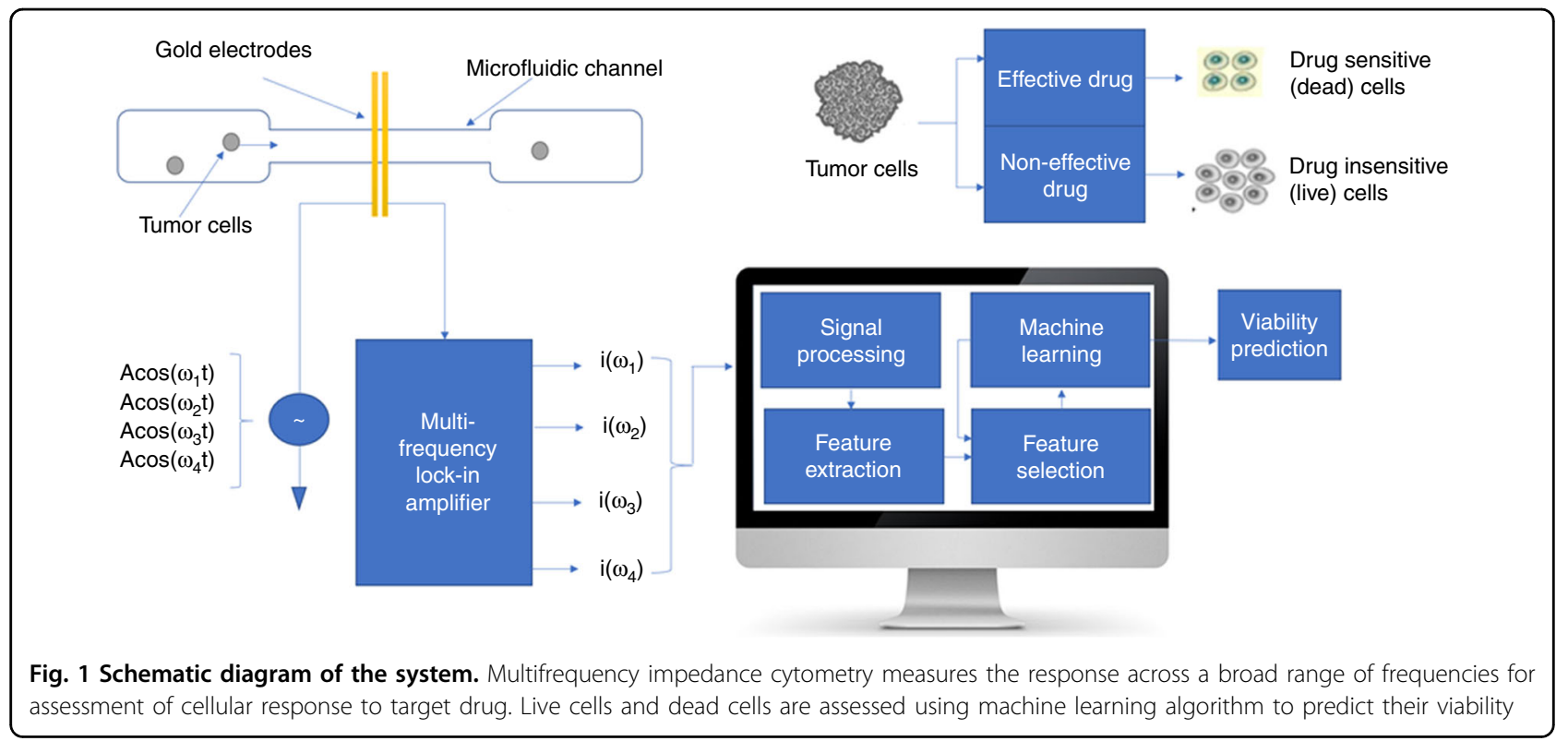

a

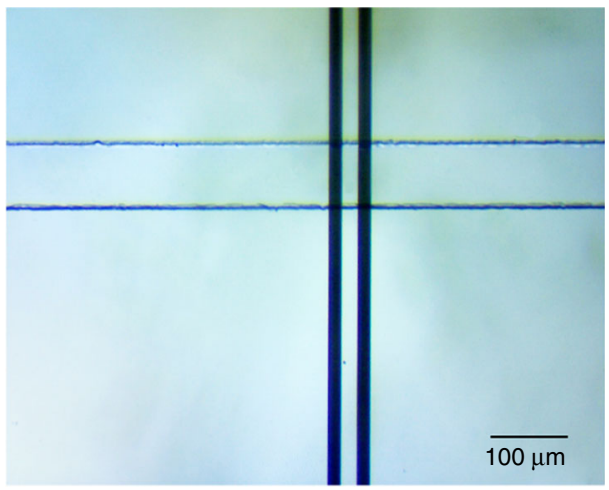

b

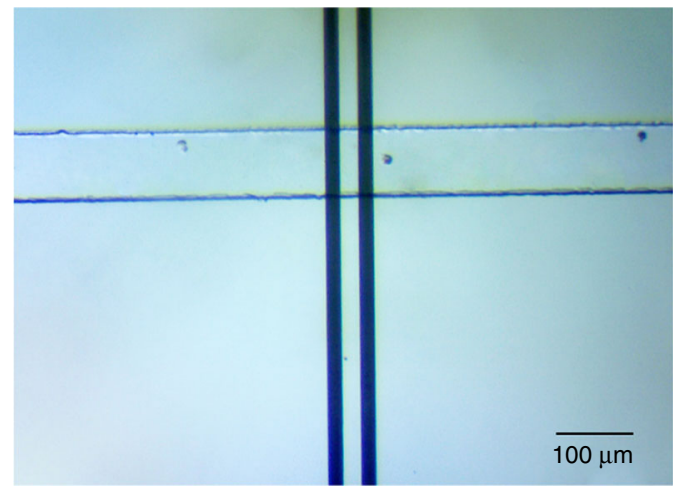

Fig. 2 Device micrograph. a Microfabricated electrodes at the channel. b Cancer cells flowing through the microfluidic channel

measurements at discrete frequencies ranging from $300 \mathrm{kHz}$ to $30 \mathrm{MHz}$. For each cell type we performed a series of measurements at four discrete frequencies simultaneously. We always used $f=500 \mathrm{kHz}$ as one of the frequencies for each set of measurements.

After the cell culture was performed, and cells were treated with drug for different durations, the cells in the media (RPMI 1640) were centrifuged ( $290 \mathrm{~g}$ for $5 \mathrm{~min}$ ) and suspended in $1 \times$ PBS $(\sim 400$ cells $/ \mu \mathrm{L})$ to perform the impedance cytometry experiments. Impedance cytometry experiments were immediately conducted after their viability was assessed using the Vi-CELL ${ }^{\mathrm{m}}$ Series Cell Viability Analyzer (Beckman Coulter, Carlsbad, CA). We performed impedance cytometry measurements for the following viability percentages: (1) $100 \%$ live cells; (2) $100 \%$ dead cells; (3) $90 \%$ live cells; $82 \%$ live cells; $50 \%$ live cells. Different viability percentages were obtained by exposing the drug to longer incubation periods.

\section{Results and discussions}

In our design, we assume an ideal polarizable electrode system with no faradic reactions as we used gold as the electrode material. When a voltage is applied across the two electrodes, it results in a double layer of ions with opposing polarity forming a boundary and acting as a capacitance, which is commonly referred to as the doublelayer capacitance. Thus, we use a simplified circuit model (Fig. 3) with a double-layer capacitance $\left(C_{d l}\right)$ at each electrode in series with the solution resistance $\left(R_{s}\right)$ in parallel with the coupling capacitance between two electrodes in the cell $\left(\mathrm{C}_{\mathrm{cell}}\right)$. Passage of cells trough the pore results in modulation of ionic resistance. The 


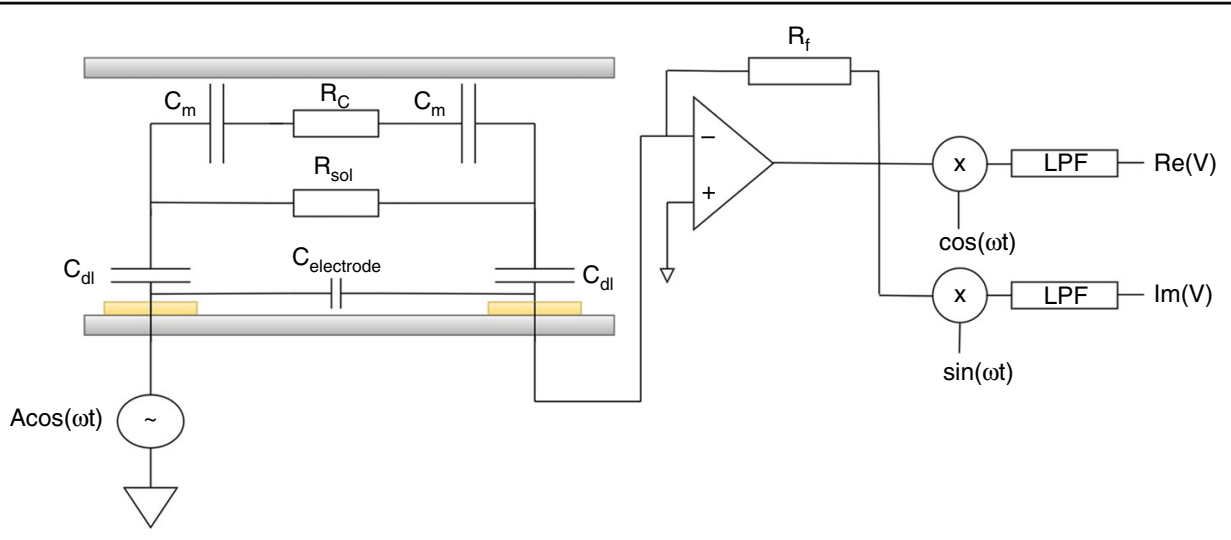

Fig. 3 Device circuit model. Equivalent circuit model of the electrode-electrolyte interface in the microchannel along with the readout circuit for measuring changes in resistance across the channel

measurements were performed using a lock in amplifier and software to record the data.

The recorded data was then post processed using an algorithm to detrend and denoise the data which helped to analyze the cytometry data with minimal error. After detrending and denoising the data we extracted two significant features from the data: amplitude change and phase change. Amplitude change was termed as change in amplitude level when a cell passes by, which is obtained by finding the difference between the baseline voltage and the peak voltage of a cell passing by. This change in amplitude was calculated for each single cell passing by with respect to its baseline for all the frequencies at which measurements were conducted. Phase change was termed as the change in angular position of the excitation frequency when a cell passes by. This was calculated from the real and imaginary data points obtained from the data. Again, change in phase was calculated for each single cell passing by for all the frequencies at which measurements were conducted.

\section{Support vector machine classifier}

SVMs are among the best "off-the-shelf" supervised learning algorithms widely used for classification and regression. In other words, when provided a labeled training data set, the algorithm will output an optimal hyperplane that can be used to classify test data. To improve the classification accuracy, we used SVM with a Gaussian Kernel. A kernel function is a form of mapping done to the training data to transform the data in higher dimensions. Mapping data in higher dimensions using a kernel function enables working with highly complex data without significant computational complexity. We used a Gaussian Kernel, which works by calculating the square of the Euclidian distance between two feature vectors. The data used for training consist of features extracted from $100 \%$ live and $100 \%$ dead cells (based on Vi-Cell data). For training the data, we labeled the features from live cells as
1 and features from dead cells as 0 . The training data set size was more than 1000 events (peaks from the impedance data corresponding to a cell passing over the electrodes) to make sure the SVM classifier does not face the problem of overfitting. To evaluate the robustness and accuracy of our SVM classifier, we tested it using three different tumor cell test samples, with differing viability percentages (90\% live, 50\% live, and $82 \%$ live). The number of 1's predicted by a classifier divided by the total number of samples gave us the viability percentage predicted by the SVM classifier.

\section{Performance of the SVM classifier}

To evaluate the performance of our SVM classifier, we used a confusion matrix on a set of test data for which the true values were known. A part of the training data, which included features from $100 \%$ live, and 100\% dead cells was used for testing. The confusion matrix was built using this data. The following performance metric was used to evaluate the SVM classifier:

$$
\text { Accuracy }=(\mathrm{TP}+\mathrm{TN}) / N
$$

TP (True Positive) is the number of the times the classifier accurately predicted the cell is live, provided the cell was actually alive, TN (True Negative) is the number of times the classifier accurately predicted the cell was dead, provided the cell was actually dead and $N$ is the total number of data points.

\section{Amplitude change as feature for classification}

We studied amplitude change at different frequencies as features for our SVM classifier. Figure 4a, b represents the normalized impedance response of live cancer cells and dead cancer cells (T47D cancer cells) at $500 \mathrm{kHz}, 20 \mathrm{MHz}$, and $30 \mathrm{MHz}$. Higher frequencies $(>10 \mathrm{MHz})$ probe the internal properties of the cell ${ }^{21}$. Figure 5 presents the spectrum of amplitude change for live and dead cancer 

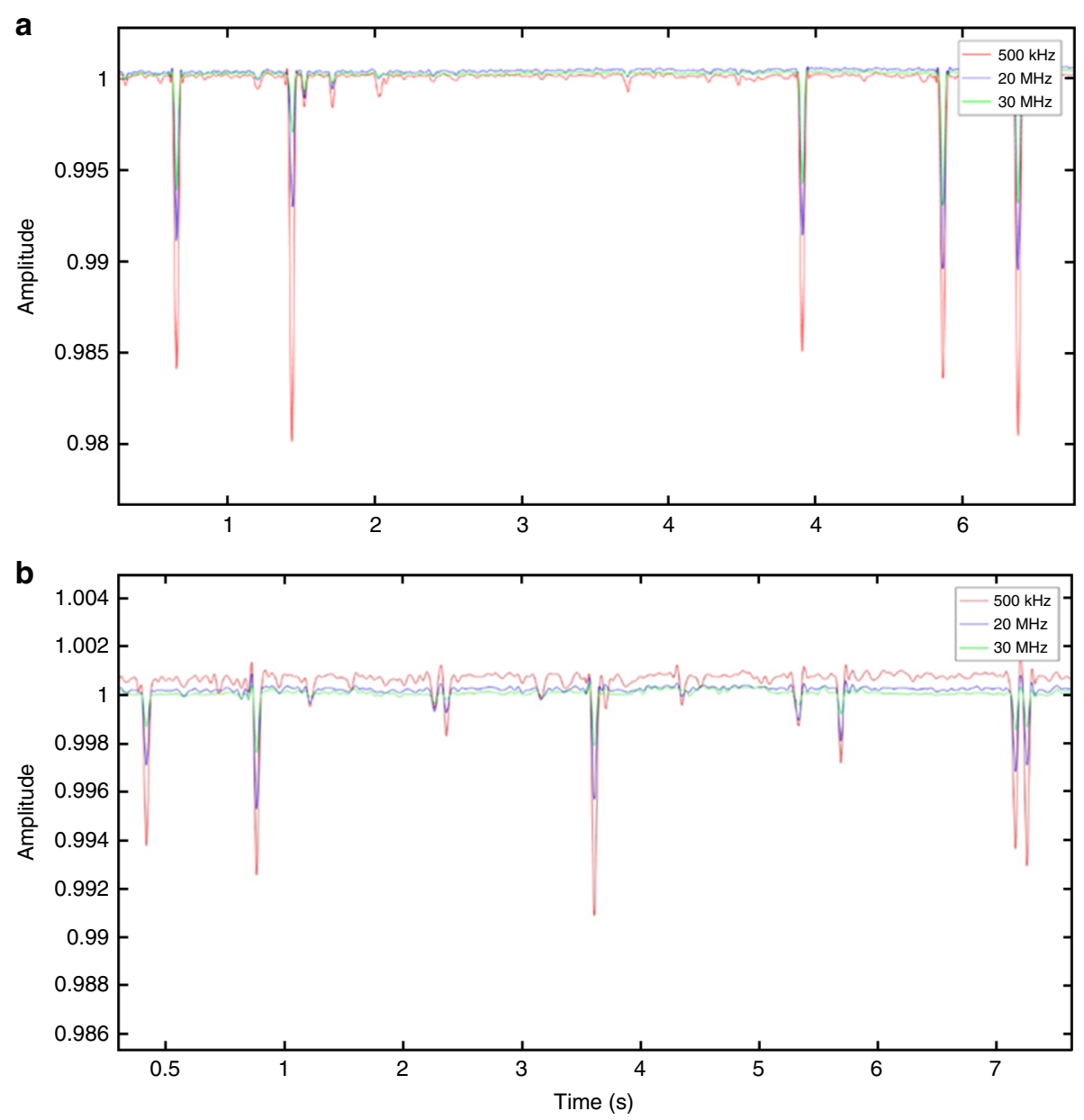

Fig. 4 Time series data of single cells. Normalized impedance response of a live cancer cells and $\mathbf{b}$ dead cancer cells at $500 \mathrm{kHz}, 20 \mathrm{MHz}$, and $30 \mathrm{MHz}$. Each peak corresponds to a single cell being detected

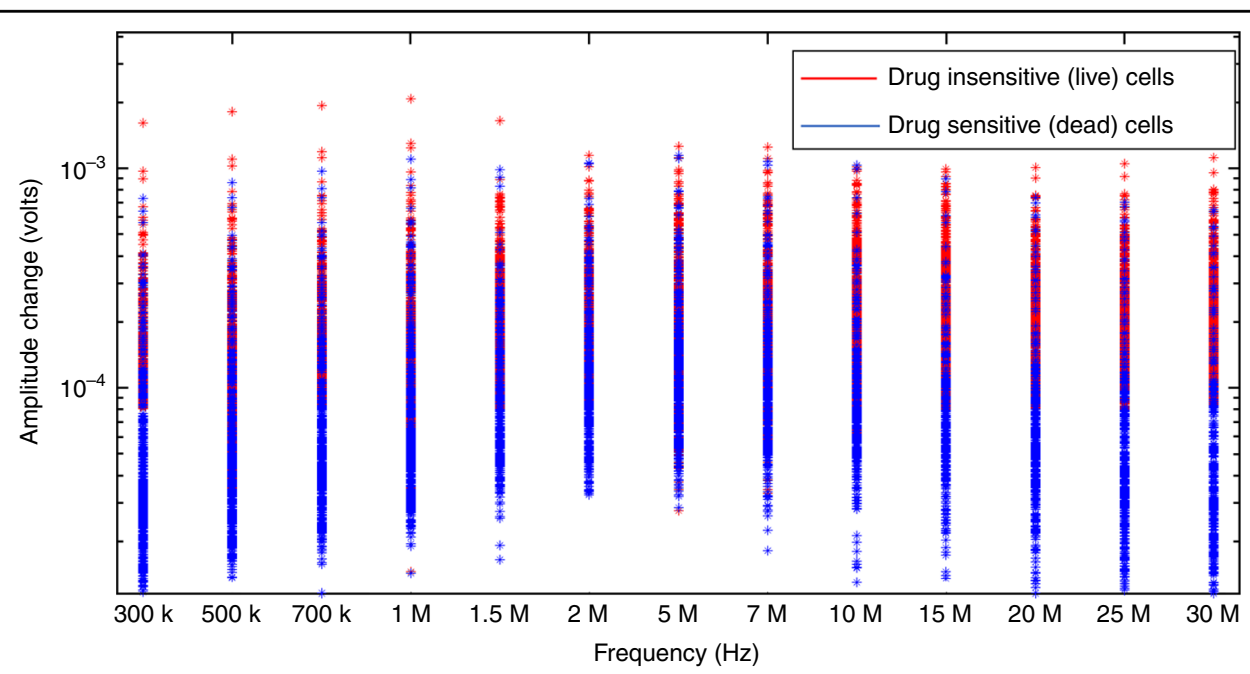

Fig. 5 Frequency dependent amplitude change. Amplitude spectrum of live cancer cells and dead cancer cells 

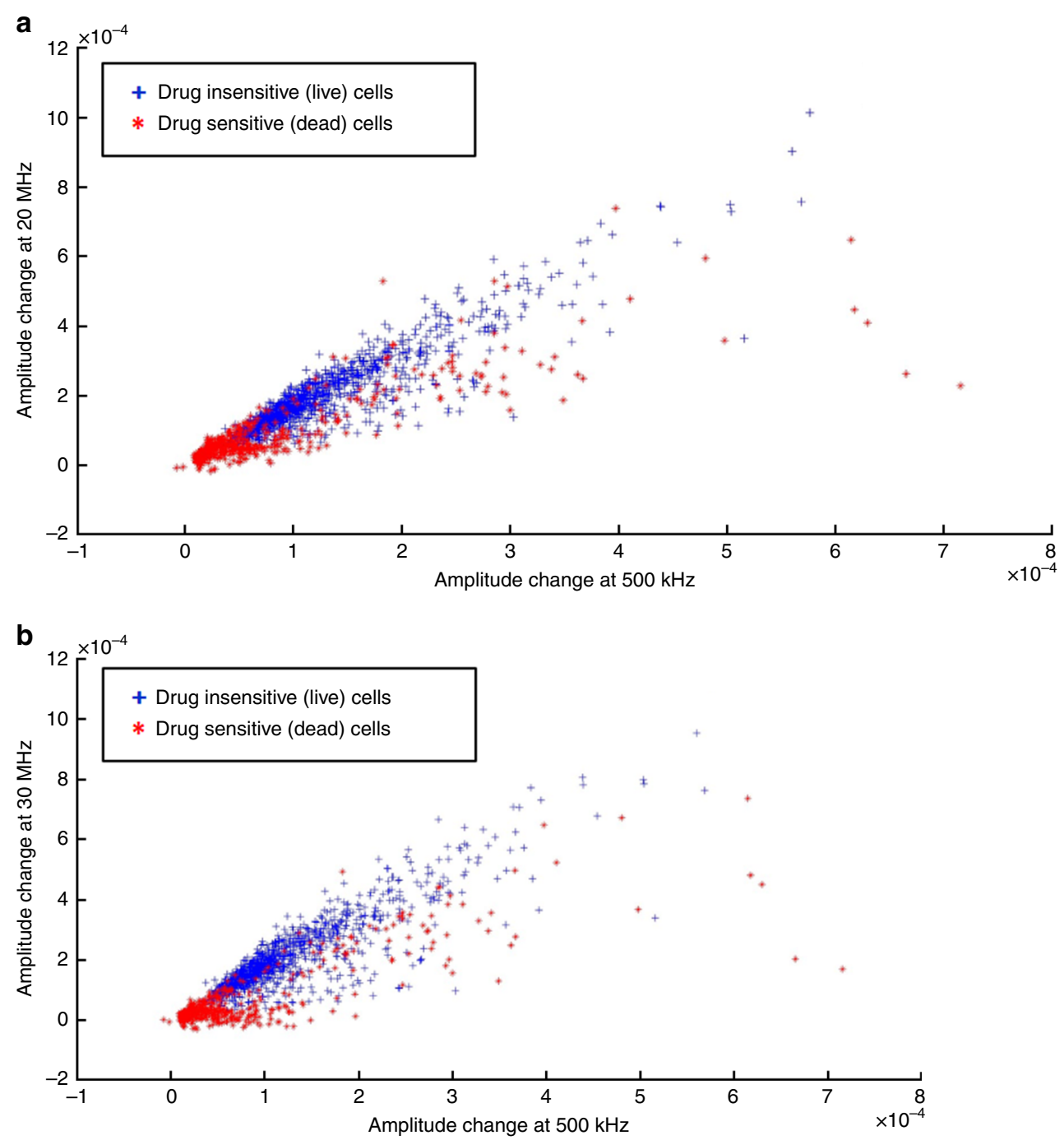

Fig. 6 Multi-frequency analysis for amplitude change. a Scatter plot of amplitude change for live cancer cells and dead cancer cells at $500 \mathrm{kHz}$ and $20 \mathrm{MHz}$. b Scatter plot of amplitude change for live cancer cells and dead cancer cells at $500 \mathrm{kHz}$ and $30 \mathrm{MHz}$

cells at a wide range of frequencies. We used amplitude change at $500 \mathrm{kHz}, 20 \mathrm{MHz}, 25 \mathrm{MHz}$, and $30 \mathrm{MHz}$ as features for our SVM classifier. Figure $6 \mathrm{a}$, b shows scatter plots representing amplitude change for live cancer cells and dead cancer cells at various frequencies. The confusion matrix for the SVM classifier using amplitude change as the feature is shown in Fig. 7a. Our classifier reported an accuracy of $89.7 \%$, with a TP and TN rate of $90 \%$ each. Figure $7 \mathrm{~b}$ represents a bar graph comparing the cell viability obtained using Trypan Blue staining (ground truth) and multifrequency impedance spectroscopy with SVM using amplitude change as features for the SVM classifier.

\section{Phase change as feature for the SVM classifier}

For phase change, we observed a general trend wherein the change (in phase) was negative at lower frequencies
$(<1 \mathrm{MHz})$ and positive at higher frequencies. Figure $8 \mathrm{a}, \mathrm{b}$ presents scatter plots representing phase change for live cancer cells and dead cancer cells (T47D cancer cells) at various frequencies. We tested our SVM classifier for all four frequency sets. The frequency set consisting of phase change at $500 \mathrm{kHz}, 20 \mathrm{MHz}, 25 \mathrm{MHz}$, and $30 \mathrm{MHz}$ reported higher accuracy for samples containing different percentages of viable cells.

The confusion matrix for the SVM classifier, using phase change as the feature is shown in Fig. 9a. Our classifier reported to have an accuracy of $90.6 \%$, a TP rate of $90 \%$ and a TN rate of $93 \%$. Figure $9 \mathrm{~b}$ represents a bar graph comparing the analysis of cell viability by Trypan Blue staining method (ground truth) and multifrequency impedance spectroscopy with SVM using phase change as features for the SVM classifier. 

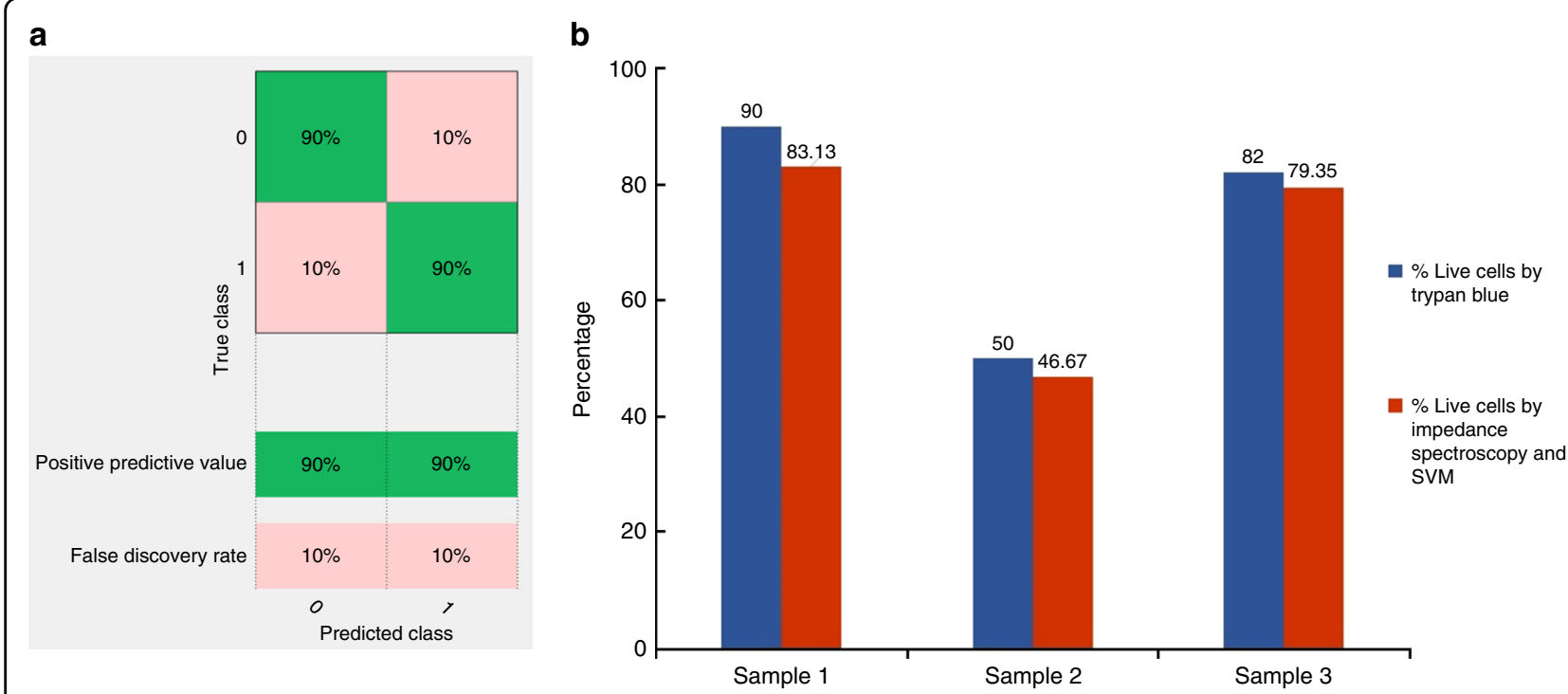

Fig. 7 Classifier accuracy when amplitude change used as features. a Confusion matrix of the SVM classifier while using amplitude change as features. $\mathbf{b}$ Comparison between the analysis of cell viability by Trypan Blue staining method (ground truth) and multifrequency impedance spectroscopy with SVM using impedance change as features for the SVM classifier

\section{Amplitude change and phase change as features for the SVM classifier}

Lastly, we explored the use of amplitude change and phase change as features for the SVM classifier. Since, for the same frequency sets $(500 \mathrm{kHz}, 20 \mathrm{MHz}, 25 \mathrm{MHz}$, and $30 \mathrm{MHz}$ ) amplitude change and phase change individually gave good results, we built an 8-feature matrix which included both amplitude change and phase change. Before training the 8-feature matrix with the SVM classifier, the data points were normalized to ensure that all the data points lie within a specified range. The confusion matrix for the SVM classifier with amplitude change and phase change as features is shown in Fig. 10a. Our classifier had an accuracy of $95.9 \%$, a TP rate of $95 \%$ and a TN rate of $97 \%$. Figure $10 \mathrm{~b}$ represents a bar graph comparing the analysis of cell viability by Trypan Blue staining (ground truth) and multifrequency impedance spectroscopy with SVM using amplitude change and phase change as features for the SVM classifier. The prediction accuracy for Sample 1 saw no improvement, compared to when only phase or amplitude change was used for training.

\section{Conclusion}

In this work we built a device capable of rapidly analyzing cell viability without staining of cells, which allows for further downstream molecular analysis. We explored the use of phase change at different frequencies as features for the SVM classifier. We found that phase change follows a trend, negative at lower frequencies and positive at higher frequencies, but the quantitative change in phase for different types of cells enables higher accuracy classification. Compared to optical techniques for labelfree analysis of cell viability, our novel method can rapidly analyze cell viability with minimal cost. We envision using this device as a point-of-care diagnostic for assessing patient response and personalization of therapeutics. Although beyond the scope of this study, we also consider ultimate practical implementation in the clinical setting. When dealing with patient samples, this drug sensitivity assay can be used for screening dissociated cells obtained from a tumor biopsy. Various protocols and kits are available commercially to dissociate cells into suspension from human tissues and tumors ${ }^{38}$. In case heterogeneity of tumor cells from patient-to-patient ends up being significant, for each test subject, it may be necessary to train the classifier on live cells from the tumor (untreated with drug) and tumor cells killed via heat shock. The trained classifier would then be capable of testing viability of cells incubated with the drug to assess efficacy. Future efforts will be dedicated toward testing with clinical samples.

\section{Materials and methods}

Electrodes are fabricated on glass using standard photolithography on a 3 " fused silica wafer. The process consists of photo-patterning resist on the fused silica wafer, electron beam metal evaporation, and liftoff processing. The process of photo-patterning includes wafer cleaning, spin coating the photoresist, soft bake of the resist, ultraviolet light exposure through a chromium mask printed on a $4^{\prime \prime} \times 4^{\prime \prime}$ glass plate, resist development, and hard bake of the resist. Following the photo- 

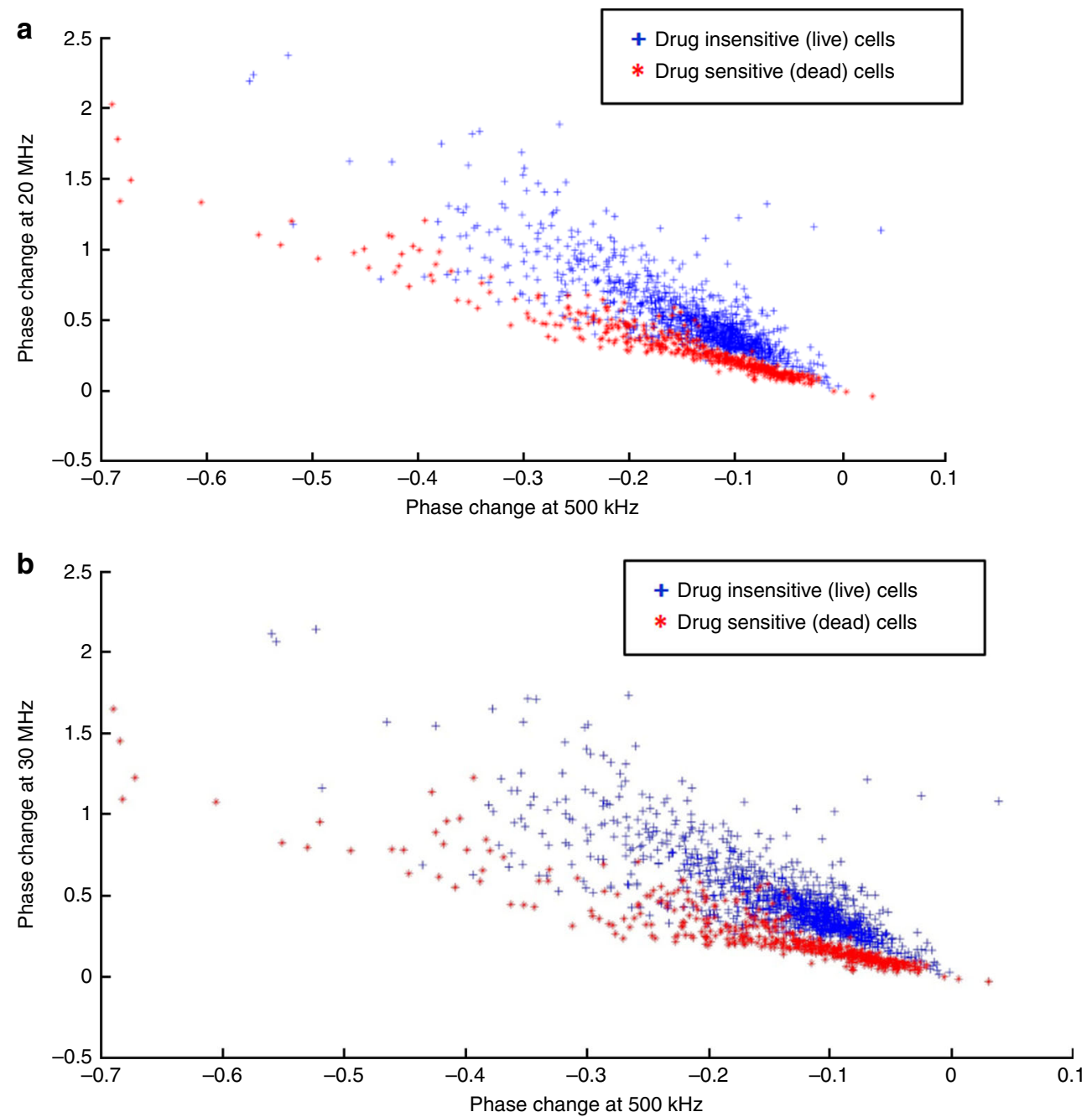

Fig. 8 Multi-frequency phase analysis. a Scatter plot of phase change for live cancer cells and dead cancer cells at $500 \mathrm{kHz}$ and $20 \mathrm{MHz}$. b Scatter plot of phase change for live cancer cells and dead cancer cells at $500 \mathrm{kHz}$ and $30 \mathrm{MHz}$

patterning process, a 100-nm-gold layer is deposited on the substrate using electron beam evaporation. A $10-\mathrm{nm}$ layer of chromium is used to enhance the adhesion of gold to the glass wafer; otherwise the gold film gets peeled off easily. We chose gold as the electrode due to its resistance to corrosion and its inert nature. The width of the electrodes was $20 \mu \mathrm{m}$ and spacing between the two electrodes was $25 \mu \mathrm{m}$.

\section{Microfluidic channel fabrication}

We fabricated the microfluidic channel itself in PDMS (Poly-dimethylsiloxane) by using soft lithography. A layer of SU-8 was patterned onto a 3" Silicon wafer that acts as a master mold. The SU-8 photo-patterning process involves standard cleaning, spin coating, soft baking, exposure, development, and hard baking. After the master mold was fabricated, PDMS (10:1 prepolymer/curing agent) was poured onto the master mold and baked at $80^{\circ} \mathrm{C}$ over $2 \mathrm{~h}$ for curing. The PDMS channel was then peeled off from the mold. A $5-\mathrm{mm}$ hole and a $1.5-\mathrm{mm}$ hole were then punched to form the inlet and outlet, respectively. The PDMS substrate was then aligned and bonded to the electrode chip after both substrates have undergone oxygen plasma treatment. The bonded chip was then baked at $70^{\circ} \mathrm{C}$ for $30 \mathrm{~min}$ to form the irreversible bond. Our microfluidic channel had a width of $100 \mu \mathrm{m}$ and height of $30 \mu \mathrm{m}$. Figure 2a represents microfabricated electrodes bonded with the channel and cancer cells flowing through the microfluidic channel as shown in Fig. 2b.

\section{Cell culture and cytotoxicity test}

T47D breast cancer cell line (in American Tissue Culture Collection also known as-HB-133) is a luminal type- 

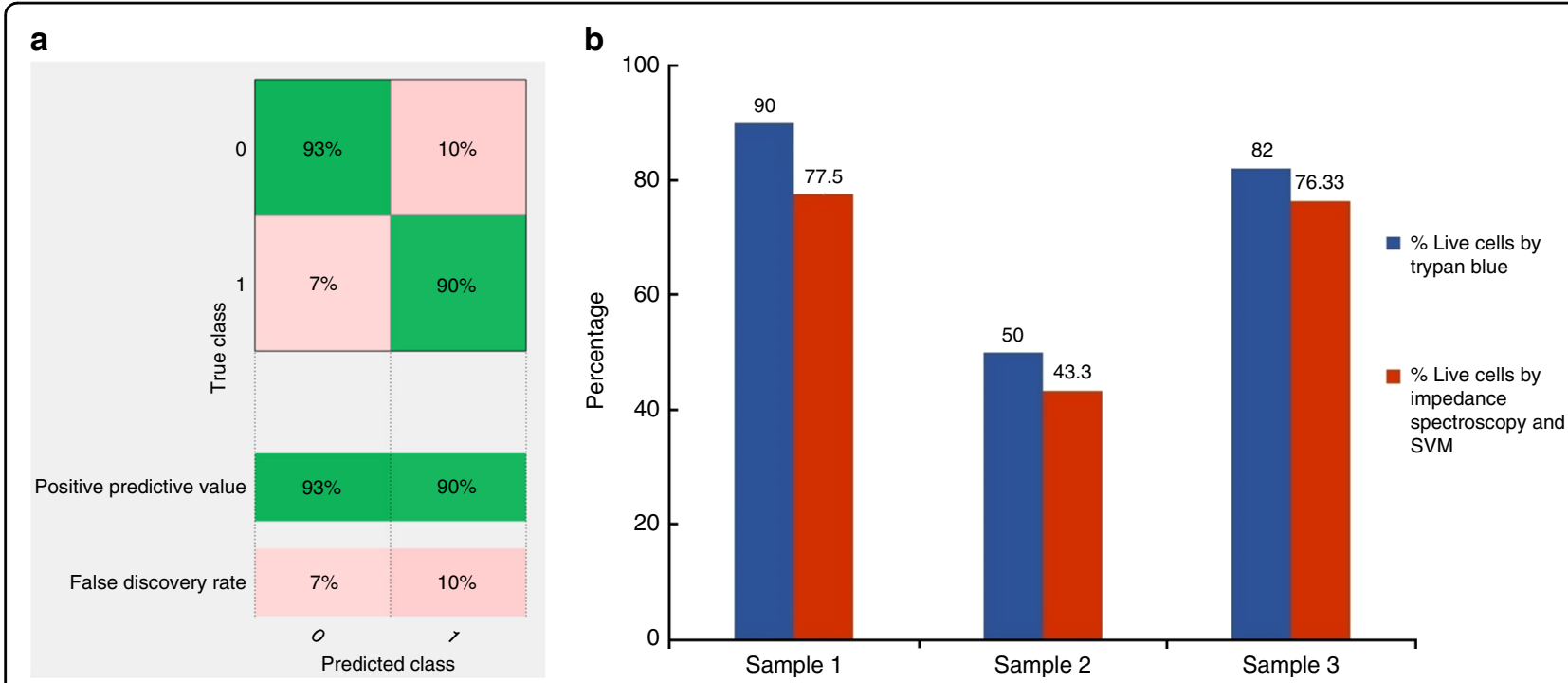

Fig. 9 Classification accuracy when phase change used as features. a Confusion matrix of the SVM classifier while using phase change as features. b Comparison between the analysis of cell viability by Trypan Blue staining method (ground truth) and multifrequency impedance spectroscopy with SVM using phase change as features for the SVM classifier
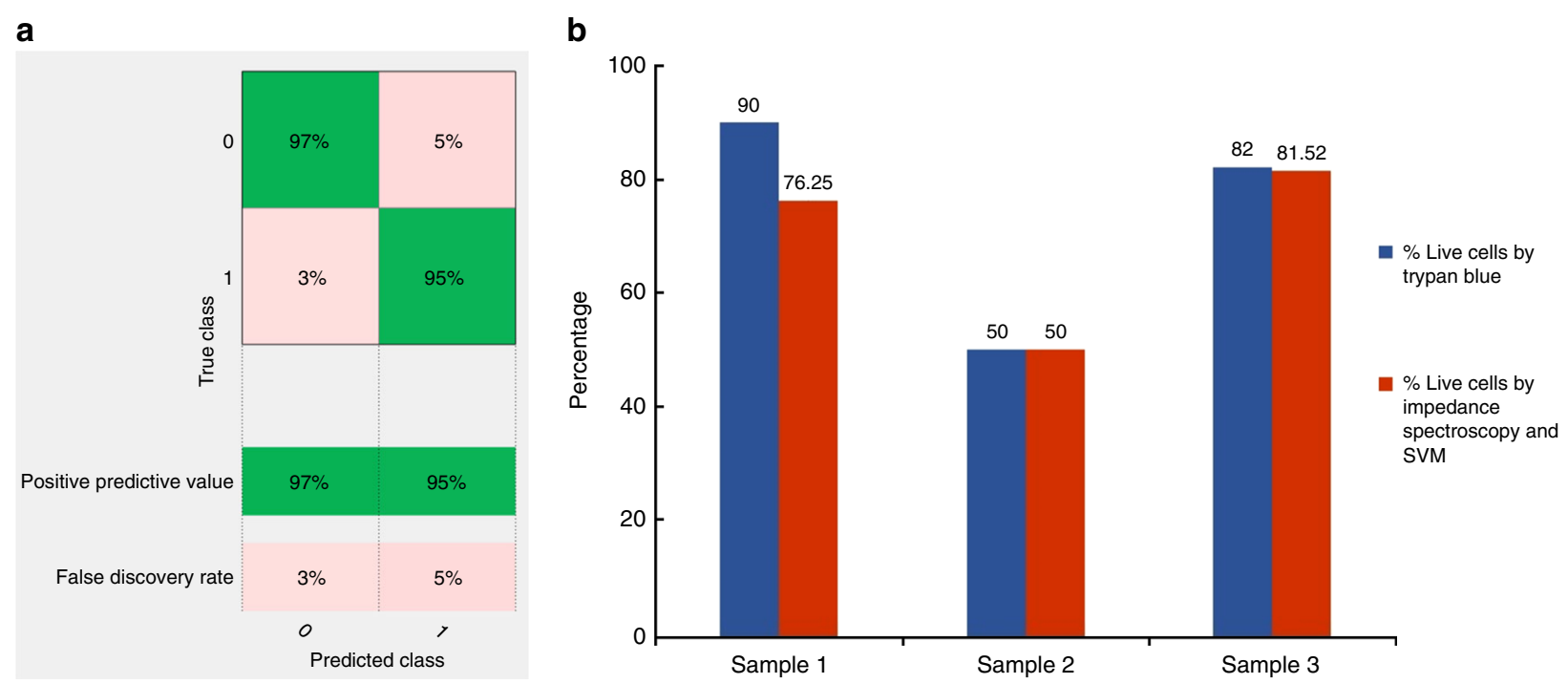

Fig. 10 Classification accuracy when both amplitude and phase change used as features. a Confusion matrix of the SVM classifier while using amplitude change and phase change as features. $\mathbf{b}$ Comparison between the analysis of cell viability by Trypan Blue staining method (ground truth) and multifrequency impedance spectroscopy with SVM using amplitude change and phase change as features for the SVM classifier

A breast cancer cell line obtained from a pleural effusion from a ductal breast cancer carcinoma patient. The cell line is also classified according to the expression of the receptors for hormonal therapy and thus classified as ER+ (Estrogen receptor positive), PR+ (Progesterone receptor positive), and HER2- (Herceptin receptor 2 negative).

For cell culture, RPMI 1640 media and fetal bovine serum albumin from Invitrogen (Fischer Scientific) were used. For the cytotoxicity assay, 5000 T47D cells per well were plated in RPMI 1640 media (Gibco) supplemented with 10\% FBS (Invitrogen). After overnight culture, spent media was removed and fresh media containing drug was added and plates were incubated for $72 \mathrm{~h}$. To assess cell viability of the T47D breast cancer cell line, at the end of the experiment the 3-(4,5-dimethylthiazol-2-yl)-5-(3-carboxymethoxyphenyl)-2-(4-sulfophenyl)-2H-tetrazolium, inner salt (MTS) assay was performed according to the 
CellTiter 96 Aqueous One Solution protocol (Promega, Madison, WI) according to manufacturer's protocol.

The cells were collected after the drug incubation time $(72 \mathrm{~h})$ and cell viability was determined using the ViCELL $^{\text {Tix }}$ Series Cell Viability Analyzer (Beckman Coulter, Carlsbad, CA). The cytotoxicity data were further analyzed using GraphPad Prism 4 software (GraphPad Software Inc., CA). The 50\% inhibitory concentration (IC50; the drug concentration required to obtain $50 \%$ cell kill compared to control) was determined using the nonlinear regression curve fit of the graphs drawn by GraphPad Prism 4 software. All experiments were performed in triplicate wells, and all experiments were repeated at least three times. The cells in logarithmic phase of growth were analyzed. T47D cells treated with our antibody-drug conjugate undergoes apoptosis, thus the cell membrane ruptures and modifies the structure of the cell, changing cell shape and diameter analyzed by the Vi-CELL analyzer.

\section{Microfluidic channel operation}

For all experiments, we relied on gravity flow to pump the fluid through the microchannel without relying on syringe pumps; given that syringe and tubing itself often introduces electronic noise and interference into the system as well. As we relied on gravity flow, this required that the channel walls be hydrophilic so that the capillary action would allow the fluid to be wicked easily and thus not impede the movement of fluid once in the channel. PDMS (Poly-dimethylsiloxane) is hydrophobic in nature, thus before every experiment we treated the bonded microchannel with oxygen plasma for $30 \mathrm{~s}$ to make the channel hydrophilic. The channel was filled with phosphate buffer saline (PBS) to preserve hydrophilicity. The difference in height resulted in a pressure difference between the inlet and the outlet, which generates flow. All measurements were performed inside a metal box to minimize external interference with the sensor and $60 \mathrm{~Hz}$ coupling.

\section{Acknowledgements}

This work was funded by the PhRMA foundation, the National Science Foundation IDBR award grant no. 1556253, and the National Science Foundation CAREER award grant no. 1846740 awarded to MJ and the Breast Cancer Research Foundation grant awarded to JRB. The authors would also like to acknowledge the Electrical and Computer Engineering department at Rutgers University. Device fabrication was performed in the Microelectronics Research Laboratory (MERL) of Rutgers University.

\section{Authors' contributions}

K.A. worked on device characterization, machine learning and performed experiments. G.M.R. performed sample preparation. J.S., Z.L., P.X., and T.L. helped with sensor fabrication. K.A. and J.S. developed code for data analysis. M.J., J.R.B., G.M.R., and K.A. performed experimental design, analyzed the data, and prepared the manuscript.

\section{Conflict of interest}

The authors declare that they have no conflict of interest.
Received: 12 August 2018 Revised: 31 January 2019 Accepted: 25 March 2019

Published online: 15 July 2019

\section{References}

1. Wagman, L. D. Principles of surgical oncology in Pazdur R., Wagman L. D., Camphausen K. A. \& Hoskins W. J. (eds) Cancer Management: A Multidisciplinary approach. 11th edn (UBM Medica, Norwalk, CT, USA, 2008).

2. Airley, R. Cancer chemotherapy: Basic science to the clinic. Churchill Livingstone; 6th edn (Wiley-Blackwell, Hoboken, NJ, 2009).

3. Bomford, C. K., Kunkler, I. H. \& Walter, J. Walter and Miller's Textbook of Radiation Therapy 6th edn, page 311.

4. Brunton, L. L. (ed.) John, S. Lazo \& Keith, L. Parker, Associate Editors. Goodman \& Gilman's The Pharmacological Basis of Therapeutics 11th edn, United States of America: The McGraw-Hill Companies, Inc. (2006).

5. NCl Dictionary of Cancer Terms. National Cancer Institute, uww.cancer.gov/ dictionary?cdrid=270742.

6. NCI Dictionary of Cancer Terms. National Cancer Institute. www.cancer.gov/ publications/dictionaries/cancer-terms.

7. Lin, S.Y., Bertino, J. R., Lin, C.-Y. Targeting tumor cells with chemotherapeutic agents conjugated to matriptase antibodies. Google patents (2016).

8. Bertino, J. R., Lin, S.-Y., Lin, C.-Y. Targeted delivery of doxorubicin conjugated with anti-matriptase antibody to treat multiple myeloma. Proceedings: AACR 101st Annual Meeting (Washington, DC, 2010).

9. van Meerloo, J., Kaspers, G. J. L. \& Cloos, J. Cell sensitivity assays: the MTT assay. In: Cree I. (eds) Cancer Cell Culture. Methods in Molecular Biology (Methods and Protocols), vol 731 (Humana Press, 2011).

10. "Cell Viability." Bioluminescent Reporters, www.promega.com/products/cellhealth-assays/cell-viability-and-cytotoxicity-assays/celltiter_glo-luminescentcell-viability-assay/ /link.aspx?_id=315FF459A2E240E8B2C0947643E0F604\&_ $\mathrm{z}=\mathrm{z}$.

11. Sawyers, C. Targeted cancer therapy. Nature 432, 294-297 (2004).

12. Miller, O. J. et al. High-resolution dose-response screening using droplet-based microfluidics. Proc. Natl Acad. Sci. USA 109, 378-383 (2012).

13. Ding, Y. et al. Microfluidic-enabled print-to-screen (P2S) platform for highthroughput screening of combinatorial chemotherapy. Anal. Chem. https:/ doi.org/10.1021/acs.analchem.5b00826 (2015).

14. Ozkumur, A. Y., Goods, B. A. \& Love, J. C. Development of a high-throughput functional screen using nanowell-assisted cell patterning. Small 11, 4643-4650 (2015).

15. Matsumoto, Y. et al.A microfluidic channel method for rapid drugsusceptibility testing of pseudomonas aeruginosa. PLOS ONE 11, e0148797 (2016).

16. Eriksson, A. et al. Drug screen in patient cells suggests quinacrine to be repositioned for treatment of acute myeloid leukemia. Blood Cancer J. 5, e307 (2015).

17. Xu, Z. Y. et al. Application of a microfluidic chip-based 3D co-culture to test drug sensitivity for individualized treatment of lung cancer. Biomaterials 34, 4109-4117 (2013).

18. Khin, Z. P. et al. A preclinical assay for chemosensitivity in multiple myeloma. Cancer Res. 74, 56-67 (2014).

19. Jonas, $\mathrm{O}$. et al. An implantable microdevice to perform high-throughput in vivo drug sensitivity testing in tumors. Sci. Transl. Med. 7, 284 ra257 (2015).

20. Ada Hang-Heng, Wong et al. Drug screening of cancer cell lines and human primary tumors using droplet microfluidics. Nat. Sci. Rep. 7, 1-12 (2017).

21. Yookyung Jung et al. Longitudinal, label-free, quantitative tracking of cell death and viability in a 3D tumor model with OCT. Nat. Sci. Rep. 6, 1-8 (2016).

22. Yang, L., Banadana, P., Bhunia, A. \& Bashir, R. Effects of dielectropheresis on growth, viability and immuno-reactivity of listeria monocytogens. J. Biol. Eng. 2, 6 (2008).

23. Mernier, G. et al. Cell viability assessment by flow cytometry using yeast as cell model. Sens. Actuators B Chem. (2009).

24. Höber, R. Eine Methode die elektrische Leitfaehigkeit im Innern von Zellen zu messen. Arch. Gesamte Physiol. 133, 237-259 (1910).

25. Höber, R. Ein zweites Verfahren die Leitfaehigkeit im Innern von Zellen ze messem. Arch. Gesamte Physiol. 148, 189-221 (1912).

26. Sun, T. \& Morgan, H. Single-cell microfluidic impedance cytometry: a review. Microfluid Nanofluid. 8, 423-443 (2010).

27. Holmes, D. et al. Leukocyte analysis and differentiation using high speed microfluidic single cell impedance cytometry. Lab Chip 9, 2881-2889 (2009). 
28. Evander, M., Ricco, A. J., Morser, J., Kovacs, G. T., Leung, L. L. \& Giovangrandi, L. Microfluidic impedance cytometer for platelet analysis. Lab Chip 13, 722-729 (2013).

29. Simon, P., Frankowski, M., Bock, N. \& Neukammer, J. Label-free whole blood cell differentiation based on multiple frequency $A C$ impedance and lightscattering analysis in a micro flow cytometer. Lab Chip 16, 2326-2338 (2016).

30. Xie, P., Cao, X., Lin, Z. \& Javanmard, M. Top-down fabrication meets bottom-up synthesis for nanoelectronic barcoding of microparticles. Lab Chip 17, 1939-1947 (2017)

31. Zhao, Y. et al. Tumor cell characterization and classification based on cellular specific membrane capacitance and cytoplasm conductivity. Biosens. Bioelectron. 57, 245-253 (2014)

32. Lin, Z., Cao, X., Xie, P., Liu, M. \& Javanmard, M.PicoMolar level detection of protein biomarkers based on electronic sizing of beadaggregates: theoretical and experimental considerations. Biomed. Microdevices. 17 119 (2015).
33. David, F., Hebeisen, M., Schade, G., Franco-Lara, E. \& Di Berardino, M. Viability and membrane potential analysis of Bacillus megaterium cells by impedance flow cytometry. Biotechnol. Bioeng. 109, 483-492 (2012).

34. Cotres, C. \& Vapnik, V. Support-vector networks. Mach. Learn. 20, 273-297 (1995).

35. Wong, S. L. et al. Combining biological networks to predict genetic interactions. Proc. Natl Acad. Sci. USA 101, 15682-15687 (2004).

36. Middendorf, M., Kundaje, A., Wiggins, C., Freund, Y. \& Leslie, C. Predicting genetic regulatory response using classification. Bioinformatics 20, i232-i240 (2004).

37. Allen, J. E. et al. JIGSAW, GeneZilla, and GlimmerHMM: puzzling out the features of human genes in the ENCODE regions. Genome Biol. 7, S9 (2006).

38. Leelatian, $\mathrm{N}$. et al. Preparing viable single cells from human tissue and tumors for cytomic analysis. Curr. Protoc. Mol. Biol. 118, 25C.1.-25C.1.23. (2017). 